\title{
Peripheral type squamous cell carcinoma of the lung: clinicopathologic characteristics in comparison to the central type
}

\author{
Yeoun Eun Sung ${ }^{1}$, Uiju Cho ${ }^{2}$, Kyo Young Lee ${ }^{1}$ \\ 'Department of Hospital Pathology, Seoul St. Mary's Hospital, College of Medicine, The Catholic University of Korea, Seoul; \\ 2Department of Hospital Pathology, St. Vincent's Hospital, College of Medicine, The Catholic University of Korea, Seoul, Korea
}

\begin{abstract}
Background: Squamous cell carcinomas (SqCCs) of the lung are known to arise more often in a central area but reports of peripheral SqCCs have increased, with a pathogenesis that is obscured. In this study, the clinicopathologic characteristics of peripheral lung SqCCs were studied and compared with those of the central type. Methods: This study included 63 peripheral lung SqCCs and 48 randomly selected central cases; hematoxylin and eosin-stained slides of surgically resected specimens were reviewed in conjunction with radiologic images and clinical history. Cytokeratin-7 immunohistochemical staining of key slides and epidermal growth factor receptor $(E G F R) / K R A S$ mutations tested by DNA sequencing were also included. Results: Stages of peripheral SqCCs were significantly lower than central SqCCs $(p=.016)$. Cystic change of the mass $(p=.007)$, presence of interstitial fibrosis $(p=0.007)$, and anthracosis $(p=.049)$ in the background lung were significantly associated with the peripheral type. Cytokeratin-7 positivity was also higher in peripheral SqCCs with cutoffs of both $10 \%$ and $50 \%(p=.011)$. Pathogenic mutations in EGFR and KRAS were observed in only one case out of the 72 evaluated. The Cox proportional hazard model indicated a significantly better disease-free survival $(p=.009)$ and the tendency of better overall survival $(p=.106)$ in the peripheral type. Conclusions: In peripheral type, lower stage is a favorable factor for survival but more frequent interstitial fibrosis and older age are unfavorable factors. Multivariate Cox analysis revealed that peripheral type is associated with better disease-free survival. The pathogenesis of peripheral lung SqCCs needs further investigation, together with consideration of the background lung conditions.
\end{abstract}

Key Words: Squamous cell carcinoma; Lung neoplasms; Peripheral; Cytokeratin-7

Received: December 24, 2019 Revised: April 10, 2020 Accepted: May 4, 2020

Corresponding Author: Kyo Young Lee, MD, Department of Hospital Pathology, Seoul St. Mary's Hospital, College of Medicine, The Catholic University of Korea, 222 Banpo-daero, Seocho-gu, Seoul 06591, Korea

Tel: +82-2-2258-1618, Fax: +82-2-2258-1627, E-mail: leekyoyo@catholic.ac.kr

Lung cancer is known to be the leading cause of cancer fatalities worldwide, in both developed and less developed countries [1]. Squamous cell carcinoma (SqCC) is one of the major nonsmall cell lung carcinomas and can be classified according to the location of the tumor into either a central or peripheral type [2]. About one-third of SqCCs are known to arise from the periphery, while the majority of SqCCs are associated with the central portion of the lung [2]. More recently, the peripheral type of SqCCs has been reported to increase, and now comprise approximately one-half of the SqCCs of the lung [3,4]. Multistage pathogenesis of SqCC arising in the central airway epithelium is known, with several sequential molecular abnormalities; starting at the normal epithelium, through squamous metaplasia, dysplasia, and carcinoma in situ [5]. Several sequential molecular abnormalities are recognized, including 3p loss of heterozygosity, $9 \mathrm{p}$ loss of heterozygosity, telomerase activation, methylation of tumor suppressor gene, and $5 \mathrm{q}$ loss of heterozygosity, that contribute to the development of invasive carcinoma [5]. On the other hand, the exact etiology of peripheral SqCCs remains unknown, with only a few studies of molecular events associated with peripheral SqCCs using animal models [6,7]. Although peripheral lung SqCCs have been investigated [3,8-10], the studies are limited in number with partly inconsistent results. In this study, we tried to reveal the clinicopathologic, immunohistochemical (cytokeratin-7), and molecular (epidermal growth factor receptor $[E G F R]$ and $K R A S)$ characteristics of the peripheral type of SqCCs, and compared them to the central type. Not only the tumor but also the background lung condition was in- 
cluded as the object of research. Furthermore, this study aimed to see if any of the findings suggested some indication of the pathogenesis or associated factors of peripheral SqCCs.

\section{MATERIALS AND METHODS}

\section{Study subjects}

Patients with surgically resected specimens of lung SqCC from January 2011 to December 2013 at Seoul St. Mary's Hospital were reviewed. Specimens of patients who had received neoadjuvant chemotherapy or radiotherapy for $\mathrm{SqCC}$ or had a previous malignancy at other sites were excluded. In total, 63 cases of peripheral SqCC, with clinical history, radiologic data, and immunohistochemical study available, were included. As well as these 63 peripheral SqCCs, 48 randomly selected non-peripheral $\mathrm{SqCCs}$ (central SqCC) were also included in this study, for comparison. Peripheral SqCC was defined as SqCC located at or distal to subsegmental bronchi [3,11], based on both radiologic images and pathologic findings. The survival length was defined as the interval in months between the day of surgical resection and the date of either the last follow-up or death. Each cause of death was reviewed to exclude death due to any other cause.

\section{Pathological studies}

The surgically resected specimens for SqCCs were fixed routinely in $10 \%$ formalin, followed by embedding in paraffin, 4-micron sectioning, and hematoxylin and eosin (H\&E) staining. The determination of histological classification and TNM classification was based on the World Health Organization classification (2015) [5]; Verhoeff-Van Gieson Elastic staining was additionally done with the cases in need for pleural status evaluation. The presence of the adenocarcinoma component was evaluated by morphological features of adenocarcinoma, in conjunction with immunohistochemical studies of thyroid transcription factor-1 (TTF-1) (Fig. 1A-C). Adenosquamous carcinoma, which requires at least $10 \%$ of both an adenocarcinoma and SqCC component, was excluded. Cystic change in the mass was defined as a definite presence of cystic space inside the mass lesion, revealed in computed tomography (CT) images and/or gross examination of the resected specimens (Fig. 1D-F). Interstitial fibrosis, including usual interstitial pneumonia (idiopathic pulmonary fibrosis) (Fig. 1G-I), emphysema, bronchiectasis, and anthracosis (Fig. 1J) were decided by characteristic histologic findings, with or without concomitant radiological evidence. The occupational history of the patients was reviewed for evaluation of pneumoconiosis, with applicable CT images and evidence of histological findings (Fig. 1K, L). History of tuberculosis was also reviewed with radiologic and/or pathologic evidence of tuberculous scars.

\section{Antibodies and immunohistochemical studies with evaluation}

Among the 111 cases, immunohistochemical studies were available for 105 cases (94.6\%); 61 peripheral SqCCs (61 out of $63,96.8 \%$ ) and 44 central SqCCs (44 out of 48, 91.7\%). Six cases were excluded due to the absence of preexisting informed consent for the further use of their human-derived materials. Immunohistochemical staining was performed with antibodies of TTF-1 (1:200, clone SPT24, Novo, Newcastle upon Tyne, UK) and cytokeratin-7 (CK7; 1:50, clone OV-TL 12/30, Dako, Glostrup, Denmark). TTF-1 analysis was integrated as part of evaluating glandular component of SqCCs, as previously described. Each glandular morphology by H\&E staining was confirmed by TTF-1 positivity. Representative sections of each SqCC were stained for $\mathrm{CK} 7$, which were interpreted and categorized as negative $(0 \%)$, focally positive $(+1,<10 \% ;+2,10 \%-50 \%)$, and positive $(+3, \geq 50 \%)$. For SqCC cases containing a focal glandular (adenocarcinoma) component, $\mathrm{CK} 7$ positivity was evaluated only in the SqCC component.

\section{Molecular studies}

Among the 111 cases, molecular studies. including assessment of $E G F R$ and KRAS, were performed in $72(64.9 \%)$ of the patients, who consented to the test performance: 44 peripheral SqCCs ( 44 out of 63, 69.8\%) and 28 central SqCCs (28 out of $48,58.3 \%)$. DNA was extracted from formalin-fixed paraffinembedded specimens by using a Maxwell 16 Tissue DNA Purification Kit (Promega, Madison, WI, USA) following the manual. Polymerase chain reaction (PCR) was performed by using Qiagen HotStar Taq DNA Polymerase (Qiagen, Hilden, Germany). The forward sequencing primers used were 5'-ACTGCTTTCCAGCATGGTGAGG-3' for EGFR exon 18, 5'-GTGGCACCATCTCACAATTGCC-3' for EGFR exon 19, 5'-ATGCGTCTTCACCTGGAAGG-3' for EGFR exon 20, 5'-CCTGAA TTCGGATGCAGAGCTTC-3' for EGFR exon 21, 5'-GGTGAGTTTGTATTAAAAGG-3' for KRAS exon 2 and 5'-GGTGCACTGTAATAATCCAGAC-3' for KRAS exon 3. PCR conditions consisted of initial denaturing at $95^{\circ} \mathrm{C}$ for 5 minutes, 40 cycles at $94^{\circ} \mathrm{C}$ for 30 seconds, at $60^{\circ} \mathrm{C}$ (EGFR exon 18) or $57^{\circ} \mathrm{C}$ (EGFR exon 19, 20, and 21) or $50^{\circ} \mathrm{C}$ (KRAS exon 2 and 3) for 30 seconds, at $72^{\circ} \mathrm{C}$ for 30 seconds and a final extension at $72^{\circ} \mathrm{C}$ for 7 minutes. The PCR products were sequenced using the BigDye Terminator v3.1 Cycle Sequencing kit (Applied 
Biosystems, Foster City, CA, USA), according to the manufacturer's instructions.

\section{Statistical analysis}

IBM SPSS ver. 22.0 (IBM Corp., Armonk, NY, USA) and R ver. 3.6.2 [12] was used for statistical analysis in this study. The correlation between each type of lung SqCC and clinicopatho-
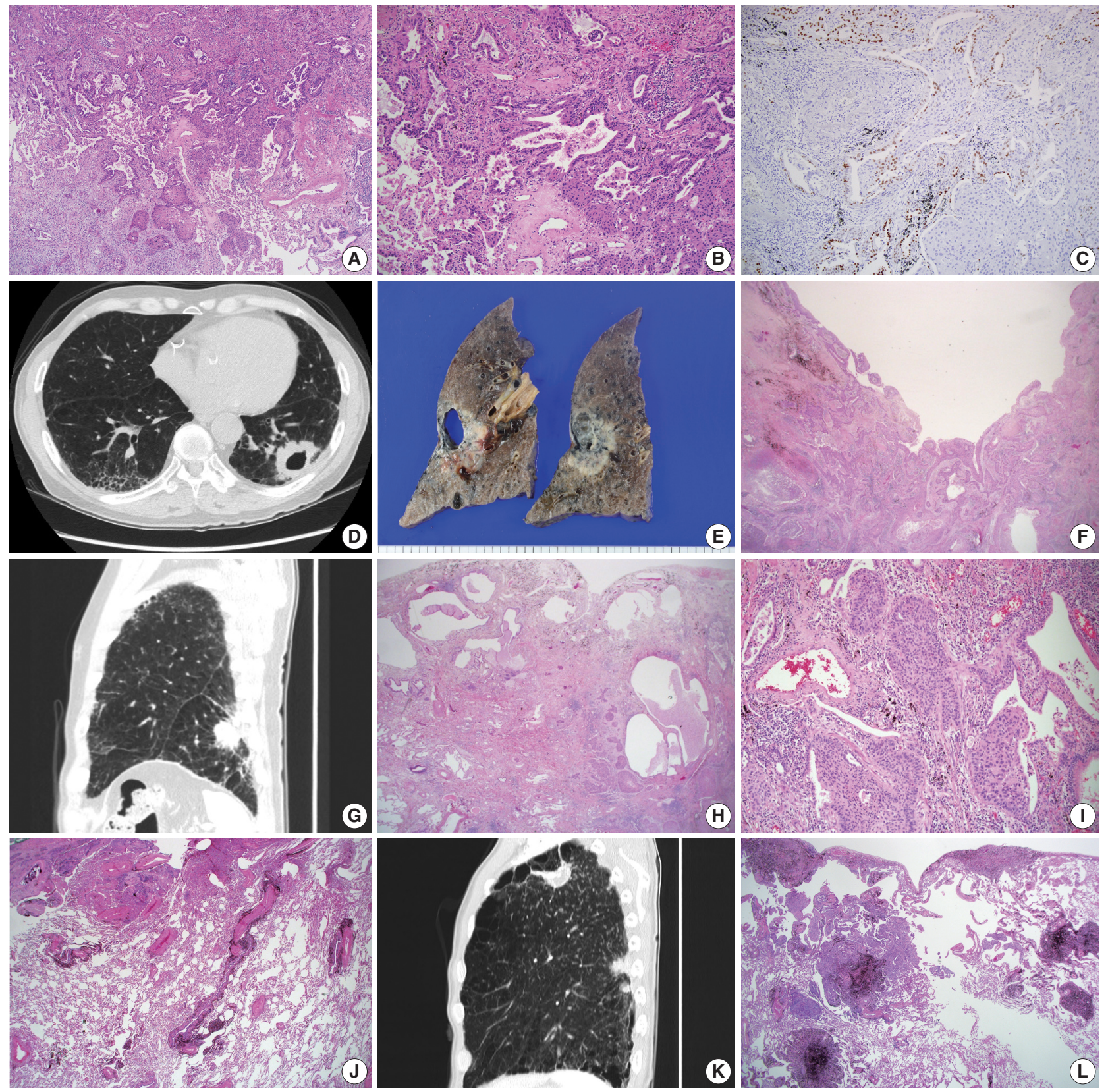

Fig. 1. Clinicopathologic characteristics of peripheral lung squamous cell carcinoma. (A-C) Microscopic findings of the glandular component (adenocarcinoma component) in peripheral squamous cell carcinoma of the lung: H\&E staining (A, B) and thyroid transcription factor-1 immunohistochemical staining (C). (D-F) Radiologic and pathologic images of cystic change of the mass: computed tomography (CT) image of the chest (D), gross examination (E), and H\&E staining (F). (G-I) Interstitial fibrosis, especially usual interstitial pneumonia with squamous cell carcinoma in the peripheral lung; CT image showing coarse reticulation with honeycombing and mass in the lower lobe of the lung (G), H\&E staining $(\mathrm{H}, \mathrm{I})$. (J) Overall anthracosis coexisting with squamous cell carcinoma in the left upper area of the image. (K, L) Peripheral squamous cell carcinoma in pneumoconiosis lung: CT image $(K)$ and H\&E staining $(L)$ showing progressive massive fibrosis with irregular mass at the subpleural portion of the left lower lobe. 
logic data was evaluated by the chi-square test for discrete variables. For variables having an expected count of less than 5, Fisher exact test was used. The continuous variables including age and smoking history (pack years) were first tested by the Shapiro-Wilk normality test, and analyzed by the Wilcoxon rank-sum test. Immunohistochemical study with $\mathrm{CK} 7$ was finally categorized as $0 \%-10 \%$ versus $\geq 10 \%$ and $<50 \%$ versus $\geq 50 \%$, both analyzed by the chi-square test. The length of survival was defined as the interval between the day of surgical resection and the date of death or the last follow-up, in months; 40 deaths occurred and the median follow-up period was 35.3 months. Survival analysis was done by Kaplan-Meier curve and
Cox proportional hazard model. Every factor and feature was analyzed individually, followed by the analysis of selected factors using the Cox proportional hazard model. In all instances, a $\leq .05$ was considered statistically significant.

\section{RESULTS}

The clinicopathologic characteristics of the 63 peripheral and 48 central lung SqCCs are summarized in Table 1. The median age of patients with peripheral or central SqCC was 70 (range, 65 to 74 ) and 68 (range, 58 to 72), respectively, with male predominance in both types $(93.7 \%$ and $97.9 \%)$. The age of pe-

Table 1. Clinicopathologic characteristics of peripheral and central squamous cell carcinoma of the lung

\begin{tabular}{|c|c|c|c|}
\hline Characteristic & Peripheral $(n=63)$ & Central $(n=48)$ & $p$-value \\
\hline Age (yr) & $70(65-74)$ & 68 (58.5-72) & $.037^{\mathrm{a}}$ \\
\hline Sex & & & .387 \\
\hline Male & $59(93.7)$ & $47(97.9)$ & \\
\hline Female & $4(6.3)$ & $1(2.1)$ & \\
\hline Smoking (pack year) & $35(20-50)$ & $40(28-50)$ & $.629^{a}$ \\
\hline T category & & & .412 \\
\hline $\mathrm{T} 1$ & 25 (39.7) & $21(43.7)$ & \\
\hline $\mathrm{T} 2$ & $31(49.2)$ & $25(52.1)$ & \\
\hline$\geq \mathrm{T} 3$ & $7(11.1)$ & $2(4.2)$ & \\
\hline $\mathrm{N}$ category & & & $<.001$ \\
\hline NO & $52(82.5)$ & $22(45.8)$ & \\
\hline$\geq \mathrm{N} 1$ & $11(17.5)$ & $26(54.2)$ & \\
\hline Stage & & & .016 \\
\hline । & $41(65.1)$ & 19 (39.6) & \\
\hline$\|$ & $18(28.6)$ & $20(41.7)$ & \\
\hline$\geq|| \mid$ & $4(6.3)$ & $9(18.8)$ & \\
\hline \multicolumn{4}{|l|}{ Pathologic features } \\
\hline Adenocarcinoma component & $6(9.5)$ & $2(4.2)$ & .462 \\
\hline Cystic change of the mass & $16(25.4)$ & $3(6.3)$ & .007 \\
\hline Interstitial fibrosis & $22(32.9)$ & $6(12.5)$ & .007 \\
\hline Emphysema & $37(58.7)$ & $24(50)$ & .360 \\
\hline Bronchiectasis & $3(4.8)$ & 0 & .257 \\
\hline Anthracosis & $35(55.6)$ & $18(37.5)$ & .049 \\
\hline Pneumoconiosis & $5(7.9)$ & $1(2.1)$ & .232 \\
\hline Tuberculosis history and/or lung scar & $18(28.6)$ & $9(18.8)$ & .232 \\
\hline Cytokeratin-7 (\%) & & & .011 \\
\hline $0-10$ & 30/61 (49.2) & $32 / 44(72.7)$ & \\
\hline $10-50$ & $12 / 61(19.7)$ & 8/44 (18.2) & \\
\hline $50-100$ & $19 / 61(31.1)$ & $4 / 44(9.1)$ & \\
\hline Metastasis and/or recur and/or death & & & $.063^{b}$ \\
\hline Event & $23(36.5)$ & $27(56.2)$ & \\
\hline Disease-free survival & $25.0(11.0-50.5)$ & $35.0(22.0-48.5)$ & \\
\hline Death & & & $.487^{b}$ \\
\hline Event & $21(33.3)$ & 19 (39.6) & \\
\hline Overall survival & $37.0(29.0-49.0)$ & $33.5(19.5-53.5)$ & \\
\hline
\end{tabular}

Values are presented as median (interquartile range) or number (\%).

Statistical analysis method: Pearson chi-square test and Fisher exact test.

aWilcoxon rank-sum test; ' Kaplan-Meier method. 
ripheral SqCC patients was significantly older than that of the central type ( $\mathrm{p}=.037)$, with no difference in sex ratio between the two groups $(\mathrm{p}=.387)$. Among the 111 patients included, 96 were smokers, with a median smoking history of 35 pack year (range, 20 to 50) in peripheral SqCC and 40 pack year (range, 28 to 50) in central SqCC. Neither the ratio of smokers to nonsmokers nor the period of smoking was associated with SqCC lung location $(\mathrm{p}=.629)$. Although $\mathrm{T}$ category did not show any difference between the two types, the frequency of lymph node metastases ( $\mathrm{N}$ category, $\geq \mathrm{N} 1$ ) was lower in the peripheral type than in the central type $(17.5 \%$ vs. $54.2 \%, \mathrm{p}<.001$; odds ratio, $0.179 ; 95 \%$ confidence interval [CI], 0.075 to 0.424 ). The overall stage evaluated by $\mathrm{T}, \mathrm{N}$, and $\mathrm{M}$ of each case showed differences between the two types, with a low-stage more frequent in patients with the peripheral type $(\mathrm{p}=.016)$.

Among the pathologic features studied, peripheral $\mathrm{SqCC}$ was associated with a more frequent cystic change of the mass (25.4\% vs. $6.3 \%, \mathrm{p}=.007$; odds ratio, $5.217 ; 95 \% \mathrm{CI}, 1.422$ to 19.140 ). For evaluation of interstitial fibrosis, not only pathologic findings but also clinical aspects were considered. Cases with definite histologic features of interstitial fibrosis showing various or uniform stages were included, but some cases with characteristic radiologic findings including honeycomb pattern were also considered to be in the 'interstitial fibrosis' group if they had consistent pathologic findings. Most of the interstitial fibrosis cases were usual interstitial pneumonia and the presence or absence of interstitial fibrosis was significantly different between the two groups (32.9\% vs. $12.5 \%, \mathrm{p}=.007$; odds ratio, $3.756 ; 95 \%$ CI, 1.382 to 10.210). Anthracosis was also more frequently found in peripheral type $\mathrm{SqCCs}(55.6 \%$ vs. 3.5\%, p = .049; odds ratio, $2.160 ; 95 \% \mathrm{CI}, 1.000$ to 4.668$)$, compared to central SqCCs. TTF-1 immunohistochemical studies were interpreted in conjunction with glandular morphologic features, to evaluate the adenocarcinoma component. The presence of the adenocarcinoma component was analyzed for each case and the difference was found to be statistically insignificant between the peripheral and central type. The remaining features, including the presence of emphysema, bronchiectasis, pneumoconiosis, and tuberculosis history were also statistically unrelated to either type of lung SqCCs.

CK7 immunohistochemical results were categorized as 0\%$10 \%, 10 \%-50 \%$, and $50 \%-100 \%$, according to the proportion of positive tumor cells out of the total number of tumor cells, as summarized in Table 1. Representative images of each proportional group are shown in Fig. 2. The proportion of each group was significantly different between peripheral and central SqCCs ( $\mathrm{p}=$ .011), with higher $\mathrm{CK} 7$ expression in peripheral SqCC. The result was the same when dividing cases of each location into two subgroups with different cutoffs; with $10 \%$ cutoff $(0 \%-10 \%$ vs. $10 \%-100 \%, \mathrm{p}=.036)$; with $50 \%$ cutoff $(0 \%-5 \%$ vs. $50 \%-100 \%$, $\mathrm{p}=.007)$. Furthermore, we analyzed if there was any correlation between $\mathrm{CK} 7$ positivity and survival in each group. For peripheral SqCCs, there existed no difference in overall survival according to $\mathrm{CK} 7$ expression $(\mathrm{p}=.912$ in the $10 \%-50 \%$ group and $\mathrm{p}=$ .915 in the $50 \%-100 \%$ group). However, for central SqCCs, higher $\mathrm{CK} 7$ positivity was associated with worse overall survival $(\mathrm{p}=.041$ in the $10 \%-50 \%$ group and $\mathrm{p}=.022$ in the $50 \%-$ $100 \%$ group).

Molecular changes in EGFR existed in four out of 44 evaluated peripheral SqCCs and four out of 28 evaluated central peripheral SqCCs; seven out of eight cases harbored silent mutations, mostly the exon $20 \mathrm{Gln} 787 \mathrm{Gln}$ (c.2361G > A) polymorphism (Table 2). Only one case (case No. 3) showed pathogenic mutation; exon 18 Glu709Gly (c.2126A > G) mutation with concomitant exon 19 Leu747_Thr751del (c.2240_2254del) (Table 2). KRAS mutation was detected in 1 out of 28 central

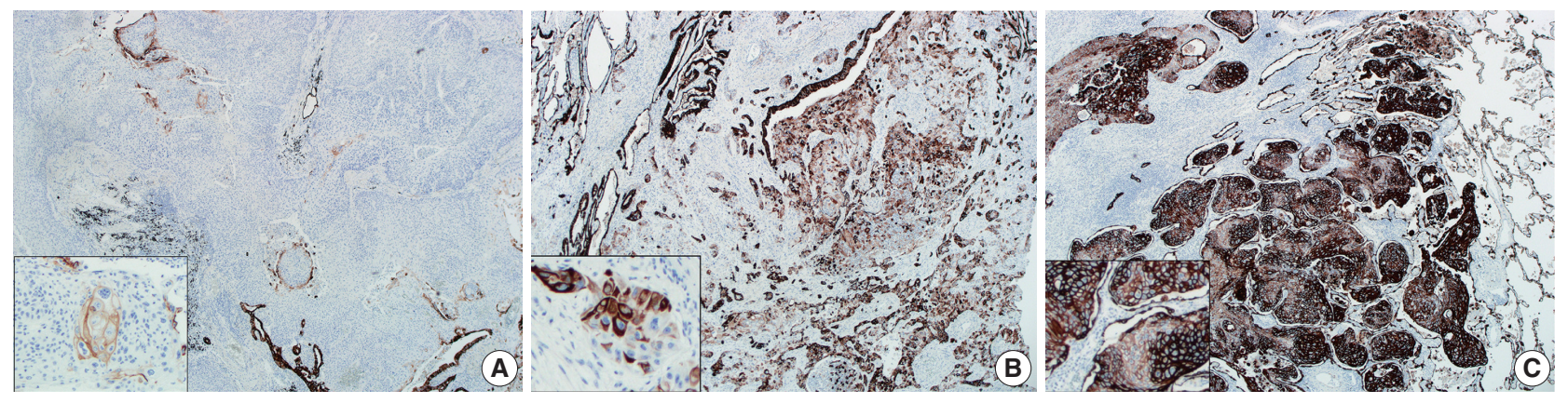

Fig. 2. Cytokeratin-7 (CK7) immunohistochemical staining of squamous cell carcinoma of the lung. (A) Less than 10\% positivity of tumor cells. Entrapped normal bronchial cells (right lower area) are excluded from the evaluation. (B) Between 10\%-50\% CK7 positivity of tumor cells, with entrapped normal bronchial cells and pneumocytes in the left and upper area. (C) More than 50\% CK7 positivity of tumor cells, with strong intensity. 
Table 2. Molecular studies for EGFR and KRAS of peripheral and central squamous cell carcinoma of the lung

\begin{tabular}{|c|c|c|}
\hline & Peripheral & Central \\
\hline \multicolumn{3}{|l|}{ EGFR mutation } \\
\hline Case No. 1 & Exon 20 Gln787Gln (c.2361G >A) polymorphism & - \\
\hline Case No. 3 & $\begin{array}{l}\text { - Exon } 18 \text { Glu709Gly (c.2126A > G) mutation } \\
\text { - Exon } 19 \text { Leu747_Thr751del (c.2240_2254del) }\end{array}$ & - \\
\hline Case No. 7 & - & Exon 20 Gln787Gln (c.2361G >A) polymorphism \\
\hline Case No. 57 & - & Exon 20 Gln787Gln (c.2361G >A) polymorphism \\
\hline Case No. 60 & - & Exon 20 Gln787Gln (c.2361G > A) polymorphism \\
\hline Case No. 61 & Exon 18 Thr725Thr (c.2175G > A) polymorphism & - \\
\hline Case No. 80 & Exon $20 \mathrm{Gln} 787 \mathrm{Gln}$ (c.2361G >A) polymorphism & - \\
\hline Case No. 97 & - & $\begin{array}{l}\text { Exon } 20 \text { Gln787GIn (c.2361G > A) polymorphism } \\
\text { Intron } 19 \text { (c.2284-60T>C) polymorphism }\end{array}$ \\
\hline \multicolumn{3}{|l|}{ KRAS mutation } \\
\hline Case No. 56 & - & Gly12Val $(c .35 G>T)$ mutation \\
\hline
\end{tabular}

EGFR, epidermal growth factor receptor.
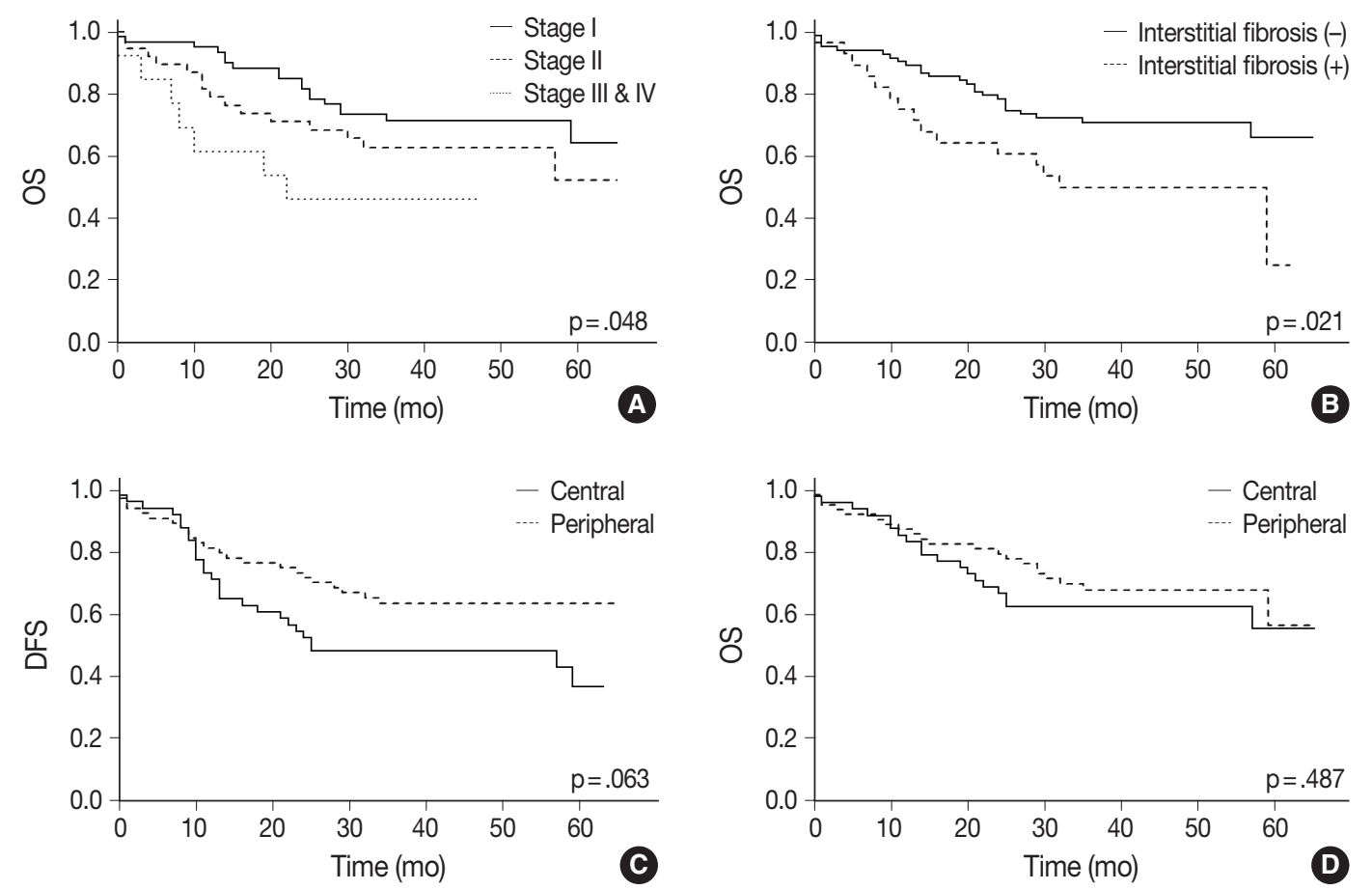

Fig. 3. Survival analysis by Kaplan-Meier curves (A) Overall survival of each stage; stage I, including stage IA and IB; sage II, including stage IIA and IIB; stage III \& IV, including IIIA, IIIB, and IV. (B) Overall survival according to the presence or absence of interstitial fibrosis; the presence of interstitial fibrosis was significantly associated with poor survival $(p=.021)$. (C, D) Disease-free survival (DFS) (C) and overall survival (OS) (D) according to the peripheral or central type of lung squamous cell carcinoma (SqCC); peripheral SqCC had a tendency of better DFS, but the result was statistically not significant $(p=.063)$; there was no significant difference in $O S(p=.487)$.

SqCCs and not found in peripheral SqCCs; the mutated case (case No. 56) harbored a Gly12Val (c.35G > T) mutation (Table 2).

A Kaplan-Meier curve of overall survival according to stages is shown in Fig. 3A. Sex and smoking history were not significantly associated with survival. Among the pathologic features listed in Table 1, only interstitial fibrosis revealed to be a significant prognosticator of overall survival; patients having interstitial fibrosis showed worse overall survival compared to those without it ( $p=.021$ ) (Fig. 3B). Patients with peripheral SqCC had a tendency of better disease-free survival (Fig. 3C) but the result was not statistically significant $(\mathrm{p}=.063)$. There was no difference in overall survival between peripheral and central lung SqCCs ( $\mathrm{p}=.487$ ) (Fig. 3D).

Based on the results obtained, the Cox proportional hazard procedure was used for further analysis. The included variables were age and stage, as well-known prognosticators, the variables 


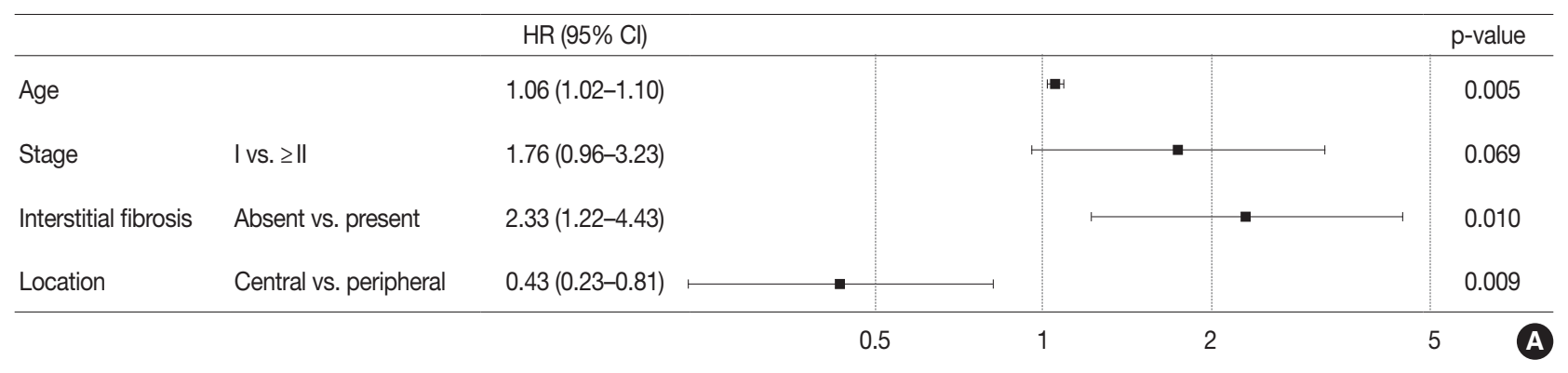

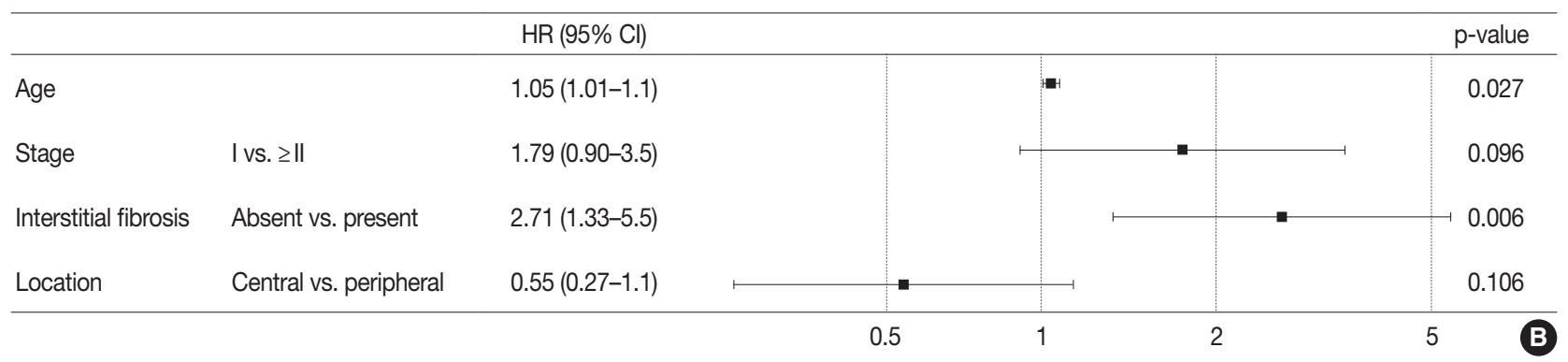

Fig. 4. Hazard ratio by Cox proportional hazard model analyzed for disease-free survival (DFS) (A) and overall survival (OS) (B). Peripheral lung squamous cell carcinoma (SqCC) was significantly associated with a better prognosis (hazard ratio [HR], 0.43; 95\% confidence interval [95\% $\mathrm{Cl}$, 0.23 to $0.81 ; p=.009)$. It also had a tendency of better OS (HR, $0.55 ; 95 \% \mathrm{Cl}, 0.27$ to 1.10$)$ but was not statistically significant $(p=.106)$.

revealed to be different between the two groups, and interstitial fibrosis. Patients with peripheral lung SqCC showed a tendency of better overall survival (hazard ratio, $0.55 ; 95 \% \mathrm{CI}, 0.27$ to 1.1) but this was not statistically significant $(\mathrm{p}=.106)$ (Fig. 4). However, in disease-free survival, peripheral lung SqCC was significantly associated with better prognosis (hazard ratio 0.43 ; 95\% CI, 0.23 to $0.81 ; \mathrm{p}=.009$ ) (Fig. 4).

\section{DISCUSSION}

In the current study, we first investigated clinicopathologic features of peripheral and central lung SqCC (Table 1). The age of patients was significantly different between the two groups $(\mathrm{p}=.037)$, which is consistent with two previous studies that found patients with peripheral lung SqCC were older $[3,10]$. T categories were not significantly different between the two types, whereas the $\mathrm{N}$ category of the peripheral type was significantly lower than the central type $(\mathrm{p}<.001)$. The tendency of lower lymph node metastases in the peripheral type was previously confirmed in one study by Funai et al. [3]; however, another study revealed no difference in metastases between the peripheral and central type [10]. In this study, the overall stage, evaluated from the $\mathrm{T}, \mathrm{N}$, and $\mathrm{M}$ category, was also different between the two types, probably due to lower lymph node metastasis in peripheral lung SqCC. The possibility cannot be ruled out that a lower $\mathrm{N}$ category, in the setting of the same $\mathrm{T}$ category, is caused by a greater physical distance from the main mass to mediastinal lymph nodes, in the peripheral SqCC.

Adenosquamous carcinoma is known to typically arise in the peripheral pulmonary parenchyma, with some cases reported to be located centrally [5]. We evaluated all the cases to determine there was a glandular component (adenocarcinoma component) in up to $10 \%$ of the tumors (Fig. 1A-C). An adenocarcinoma component was observed in $9.5 \%$ (6 out of 63 cases) and $4.2 \%$ ( 2 out of 48 cases) in peripheral and central lung SqCCs, respectively (Table 1). However, statistical analysis with Fisher's exact test revealed no significant difference, probably due to the limited number of cases.

There existed more frequent cystic change of the mass in peripheral type SqCCs than in central type (25.4\% vs. $6.3 \%$, p = .007 ), which was confirmed by both CT images and gross examination, along with microscopic findings in each case (Fig. 1D-F). Cystic change of lung cancers has been studied by several researchers of radiology $[13,14]$. The majority of the data consisted of adenocarcinoma. The cystic lesions were considered to include emphysematous bullae, congenital or fibrotic cysts, bronchiectatic airways, and distended distal airspaces [13]. In the current study, microscopic findings of the masses with cystic change revealed an empty space lined by SqCC cells or partly suspicious for $\mathrm{SqCC}$ in situ (Fig. 1F). With gross examination and CT images of some cases, the cystic lesion was suspected to have been previously bronchiolar airway, of which the proximal portion was 
blocked by a mass effect. Cystic change due to necrosis was scarce in this study. It is possible to assume that the lower proportion of cystic change in central lung SqCCs might be due to a relatively larger airway diameter obstructed by the mass. It might be noteworthy that differential diagnoses of solid masses with cystic change in the lung should include SqCC, especially those located in the peripheral area of the lung.

In the investigation of pathologic features of background lung, we focused on the diffuse and overall change of lung, in conjunction with CT images. Among those reviewed, the presence of interstitial fibrosis (Fig. 1G-I) and anthracosis (Fig. 1J) were more associated with the peripheral type than the central type of SqCCs (32.9\% vs. $12.5 \%, \mathrm{p}=.007 ; 55.6 \%$ vs. $37.5 \%, \mathrm{p}=$ .049) (Table 1). Interstitial fibrosis cases included in this study were mostly of the usual interstitial pneumonia pattern. With the established multistage pathogenesis of SqCCs, which includes morphological changes from normal epithelium through to squamous metaplasia, dysplasia, and carcinoma in situ in the central bronchial area [5], it is possible to assume that similar pathogenic events may occur in the peripheral area of the lung parenchyma if it has undergone bronchial metaplasia. In the peripheral SqCC cases with simultaneous interstitial fibrosis, SqCCs usually coexisted with interstitial fibrosis and bronchial metaplasia (Fig. 1H, I), although the sequential morphologic change from dysplasia or carcinoma in situ to $\mathrm{SqCC}$ was not sufficient to be verified microscopically.

Some authors have proposed several growth patterns for the peripheral SqCC of the lung, including pushing patterns, infiltrative patterns, alveolar filling patterns, and pseudoalveolar filling patterns [15] or an alveolar space-filling type, expanding type, and combined type [3]. These previous studies suggest that the alveolar filling growth pattern might be associated with a better prognosis [3,15]. However, in the current study, it seemed reasonable to consider the presence of diffuse interstitial fibrosis, including usual interstitial pneumonia, in evaluating peripheral SqCC of the lung for the following two reasons: (1) interstitial fibrosis might be more frequently associated with the peripheral type of SqCC than the central type and (2) for the cases of background fibrotic lung, certain growth patterns such as the alveolar filling pattern may be impossible.

In regards to the background condition of the lung, we also investigated the presence of emphysema, bronchiectasis, pneumoconiosis, and histologic evidence of past tuberculosis with or without a clinical history of tuberculosis, all of which revealed to be of no significant relevance with either type of lung SqCC (Table 1). Among these, the presence of emphysema, which was reported to be relevant to peripheral type SqCC in one previous study [9], did not show significant association in the current study $(\mathrm{p}=.360)$. Especially, patients with pneumoconiosis whose lungs show multiple scattered fibrotic nodules were hypothesized initially to have an association with peripheral type SqCCs, as interstitial fibrosis or anthracosis. There were five pneumoconiosis cases out of 63 peripheral SqCCs $(7.9 \%)$ and one case out of 48 central SqCCs (2.1\%). The statistical results may be due to the limited number of pneumoconiosis cases included as well as rare epidemiology. A further large-scale study with a greater number of patients might be necessary.

CK7 expression is usually considered a characteristic feature of adenocarcinoma of various origin sites, including lung, biliary tract, pancreas, salivary gland, breast, ovary, and endometrium [16-18]. Relatively less studied in SqCC, CK7 is known to be positive in more than $20 \%$ of lung SqCCs according to a previous study [19]. In esophageal SqCCs, a group of researchers revealed an association between $\mathrm{CK} 7$ expression and poor prognosis [20]. No such study was found for the prognostic significance of $\mathrm{CK} 7$ positivity in lung SqCC. However, the difference in immunohistochemical staining between the peripheral and central type of lung SqCCs was included in a few previous studies; CK7 expression was more frequently observed in the peripheral type in one study [10], while there was no difference between the two types in another study [9]. In both studies, tissue microarrays were evaluated. In the current study, whole slides of each key block in all tumors were stained with $\mathrm{CK} 7$ and overall a heterogenous staining pattern was obtained. Therefore, we interpreted CK7 staining with categorization according to the proportion of positive tumor cells out of the entire number of tumor cells as $0 \%-10 \%, 10 \%-50 \%$, and $50 \%-100 \%$ (Fig. 2). With cutoffs of both $10 \%$ and $50 \%$, significantly more $\mathrm{CK} 7$ positivity in peripheral type lung SqCC was confirmed (Table 1). It was notable that with a cutoff of $10 \%, 47.5 \%$ of peripheral type lung $\mathrm{SqCC}$ showed $\mathrm{CK} 7$ positivity.

As $\mathrm{CK} 7$ expression was evaluated in only SqCC, even in the cases containing a focal adenocarcinoma component, the difference of $\mathrm{CK} 7$ expression between peripheral and central groups was not associated with the glandular component. Additionally, the presence of the focal adenocarcinoma component was not significantly different between the two groups, as shown in Table 1. CK7 expression was observed not only in various percentages but also with various intensity, as shown in Fig. 2. The fact that adenosquamous carcinoma frequently arises in the peripheral area [5] and that an adenocarcinoma component of up to $10 \%$ was observed in a proportion of peripheral SqCC in this study, 
although not significant, suggests some association. A weak to moderate, and even strong, expression of $\mathrm{CK} 7$ in peripheral SqCC might suggest a different biologic and/or molecular aspect from central SqCC. This possible difference needs further study and probably needs to be separated from the cases accompanying interstitial fibrosis. Furthermore, the survival analysis according to $\mathrm{CK} 7$ expression in each location revealed high $\mathrm{CK} 7$ expression was associated with worse overall survival in central SqCC, with no survival difference in peripheral SqCC. Although this finding might suggest a new prognostic aspect of $\mathrm{CK} 7$ in central SqCC, it is limited by a relatively small number of samples (44 central SqCCs) and the fact that there was no difference in disease-free survival in both central and peripheral SqCCs.

According to previous studies, the EGFR and KRAS mutation in lung $\mathrm{SqCC}$ is sparse and reported in less than $5 \%$ of all patients [5]. In the current study, though some polymorphism was observed in several cases, only one case in the peripheral type harbored the EGFR mutation and another case in the central type had a KRAS mutation (Table 2). Until now, the molecular difference between the two types of SqCC has not been studied. This study has limitations in that only a portion of the cases collected were the object of molecular tests. For further investigation, studies with a much larger number of cases might be necessary, opening the possibility of assessing novel molecular events of peripheral type SqCCs of the lung other than EGFR or KRAS mutations.

With Kaplan-Meier curves, peripheral lung SqCC did not show a significant difference in survival, although the slight tendency of a better DFS was suggested (Fig. 3C, D). We hypothesized that a lower TNM stage in peripheral SqCC is a favorable factor, but more frequent interstitial fibrosis and older age are counteracting factors. This could be verified by the Cox proportional hazard model, which revealed an association with better survival after adjustment for age, stage, and the presence of interstitial fibrosis (Fig. 4).

In conclusion, the current study suggests that peripheral type lung SqCC is possibly different from the central type in clinicopathologic aspects, with lower lymph node metastasis but more frequently accompanying interstitial fibrosis and anthracosis in the background lung. In addition, a cystic change in the mass is more commonly observed in the peripheral type, which can be evaluated by CT images preoperatively and also with gross examination after surgery. Immunohistochemically, the peripheral type shows more tendency of $\mathrm{CK} 7$ staining, which has been known to be positive in only a minority of SqCC. Some of the cases in our study revealed rare EGFR and KRAS mutations in both types.
The peripheral type of lung SqCCs may have different pathogenic events from that of the central type, which needs further investigation with a larger set of cases. We believe the current study could be considered with further investigation and expect the information to be reflected eventually in patient care.

\section{Ethics Statement}

All procedures performed in the current study were approved by the Institutional Review Boards (IRB) of the Catholic Medical Center Office of Human Research Protection Program (KC15SISI0146) in accordance with the 1964 Helsinki declaration and its later amendments. Formal written informed consent was not required with a waiver by the appropriate IRB and/ or national research ethics committee.

\section{ORCID}

Yeoun Eun Sung https://orcid.org/0000-0002-9408-0085 Uiju Cho https://orcid.org/0000-0002-6229-8418 Kyo Young Lee https://orcid.org/0000-0001-9954-8583

\section{Author Contributions}

Conceptualization: KYL. Data curation: UC, YES. Formal analysis: UC, YES. Investigation: UC, YES, KYL. Methodology: KYL. Project administration: UC, YES. Resources: UC, YES. Supervision: KYL. Validation: KYL. Visualization: YES. Writing—original draft: YES. Writing-review \& editing: UC, YES, KYL. Approval of final manuscript: all authors.

\section{Conflicts of Interest}

The authors declare that they have no potential conflicts of interest.

\section{Funding Statement}

No funding to declare.

\section{References}

1. Torre LA, Bray F, Siegel RL, Ferlay J, Lortet-Tieulent J, Jemal A. Global cancer statistics, 2012. CA Cancer J Clin 2015; 65: 87-108.

2. Colby TV, Koss KM, Travis WD. Tumors of the lower respiratory tract, fascicle 13. Washington, DC: Armed Forces Institute of Pathology, 1995.

3. Funai K, Yokose T, Ishii G, et al. Clinicopathologic characteristics of peripheral squamous cell carcinoma of the lung. Am J Surg Pathol 2003; 27: 978-84.

4. Brooks DR, Austin JH, Heelan RT, et al. Influence of type of cigarette on peripheral versus central lung cancer. Cancer Epidemiol Biomarkers Prev 2005; 14: 576-81.

5. Travis WD, Brambilla E, Burke AP, Marx A, Nicholson AG. WHO classification of tumours of the lung, pleura, thymus and heart. Lyon: International Agency for Research on Cancer (IARC), 2015.

6. Yamano S, Gi M, Tago Y, et al. Role of deltaNp63(pos)CD44v(pos) cells in the development of $\mathrm{N}$-nitroso-tris-chloroethylurea-induced peripheral-type mouse lung squamous cell carcinomas. Cancer Sci 2016; 107: 123-32.

7. Dai YB, Miao YF, Wu WF, et al. Ablation of Liver X receptors alpha and beta leads to spontaneous peripheral squamous cell lung cancer in mice. Proc Natl Acad Sci U S A 2016; 113: 7614-9.

8. Sakurai H, Asamura H, Watanabe S, Suzuki K, Tsuchiya R. Clinicopathologic features of peripheral squamous cell carcinoma of the lung. Ann Thorac Surg 2004; 78: 222-7. 
9. Hayashi T, Sano H, Egashira R, et al. Difference of morphology and immunophenotype between central and peripheral squamous cell carcinomas of the lung. Biomed Res Int 2013; 2013: 157838.

10. Saijo T, Ishii G, Nagai K, et al. Differences in clinicopathological and biological features between central-type and peripheral-type squamous cell carcinoma of the lung. Lung Cancer 2006; 52: 37-45.

11. Shimosato Y, Suzuki A, Hashimoto T, et al. Prognostic implications of fibrotic focus (scar) in small peripheral lung cancers. Am J Surg Pathol 1980; 4: 365-73.

12. R Core Team. R: a language and environment for statistical computing [Internet]. Vienna: R Foundation for Statistical Computing, 2018 [cited 2019 Dec 24]. Available from: https://www.R-project.org/.

13. Sheard S, Moser J, Sayer C, Stefanidis K, Devaraj A, Vlahos I. Lung cancers associated with cystic airspaces: underrecognized features of early disease. Radiographics 2018; 38: 704-17.

14. Mascalchi M, Attina D, Bertelli E, et al. Lung cancer associated with cystic airspaces. J Comput Assist Tomogr 2015; 39: 102-8.

15. Yousem SA. Peripheral squamous cell carcinoma of lung: patterns of growth with particular focus on airspace filling. Hum Pathol 2009; 40: 861-7.

16. van de Molengraft FJ, van Niekerk CC, Jap PH, Poels LG. OV-TL $12 / 30$ (keratin 7 antibody) is a marker of glandular differentiation in lung cancer. Histopathology 1993; 22: 35-8.

17. Baars JH, De Ruijter JL, Smedts F, et al. The applicability of a keratin 7 monoclonal antibody in routinely Papanicolaou-stained cytologic specimens for the differential diagnosis of carcinomas. Am J Clin Pathol 1994; 101: 257-61.

18. van Niekerk CC, Jap PH, Ramaekers FC, van de Molengraft F, Poels LG. Immunohistochemical demonstration of keratin 7 in routinely fixed paraffin-embedded human tissues. J Pathol 1991; 165: 145-52.

19. Johansson L. Histopathologic classification of lung cancer: relevance of cytokeratin and TTF-1 immunophenotyping. Ann Diagn Pathol 2004; 8: 259-67.

20. Xu Y, Wang W, Li L, et al. FOXA1 and CK7 expression in esophageal squamous cell carcinoma and its prognostic significance. Neoplasma 2018; 65: 469-76. 